\title{
The ultrasound scan is not superior to the landmarks technique for performing regular lumbar regional anesthesia.
}

\section{G. Kotsovolis, E. Hatzopoulos}

Department of Anesthesia, 424 Army General Hospital - Thessaloniki (Greece)

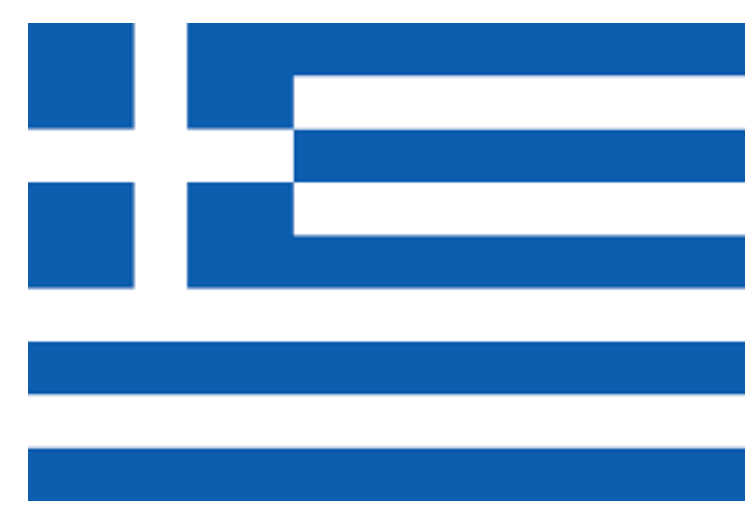

Background and Goal of Study: The ultrasound scan of the lumbar spine can potentially facilitate the performance of neuraxial anesthesia. The main purpose of the study was to determine if the ultrasound scan (US) is superior to the landmarks (LM) technique for performing every-day spinal, epidural and combined spinal-epidural anesthesia.

Materials and Methods: A prospective randomized controlled trial was designed. 1 tertiary hospital from September 2015 until September 2017 participated. The 18-80 years old ASA 1-3 patients eligible for surgery under spinal, epidural, combined spinal-epidural anesthesia or general anesthesia with epidural catheter for postoperative analgesia were included in the study. Patients with a history of lumbar spine surgery were excluded. The patients were randomly allocated to two groups: the US (the puncture site was determined by lumbar ultrasound scan) and the LM group (the puncture site was determined by the landmarks technique). Data from 146 patients were finally analyzed.

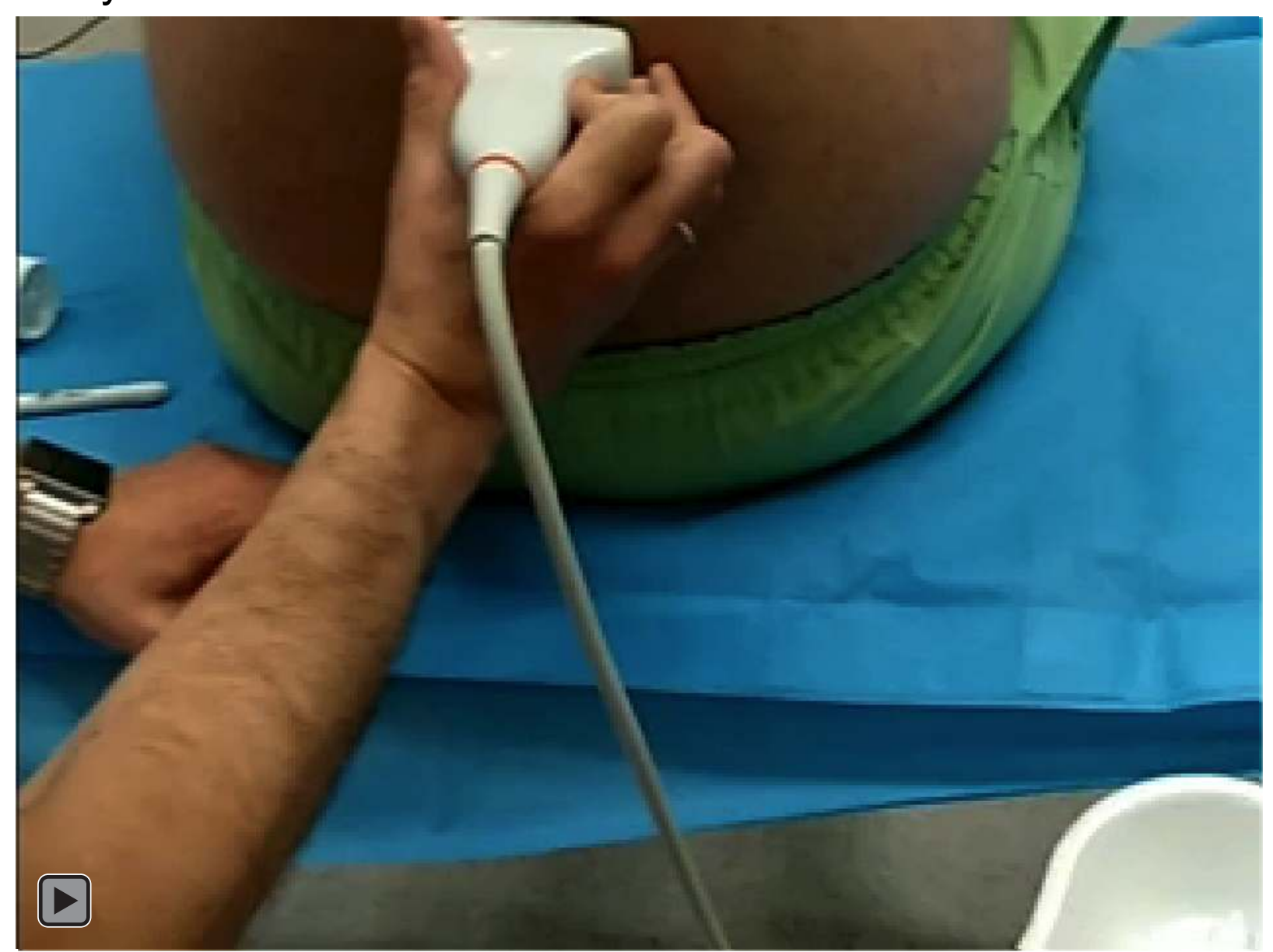

The main outcome was the single-injection success rate. Secondary outcomes were the success of the technique rate, the number of attempts and the time required to complete the technique, the number of times required to change the trajectory of the needle, the rate of change of the level of the puncture and the patient's satisfaction.
Results and Discussion: There were no differences between the US and the LM in single-puncture success rate $(60 \%$ vs $53 \%)$, success of the technique rate (95\% vs $98 \%)$ and rate of change of puncture level $(17,5 \%$ vs $30 \%)$. No differences were also found in the number of punctures per patient $(1,65$ vs 2,06$)$ and the number of times required to change the trajectory of the needle $(0,74$ vs 1,14$)$. No differences were found in patient satisfaction ( $96 \%$ vs $98 \%$ ). The only statistically but not clinically significant difference between the two groups was in the total time required to complete the technique ( 9 minutes and 10 seconds vs 7 minutes and 31 seconds, $p<0,05)$.

Conclusion(s): The US of the lumbar spine for performing regular regional anesthesia requires additional time than the LM technique without offering any advantages. Further studies are needed to examine potential advantages in special groups of patients like the obese or the patients with spinal pathology.

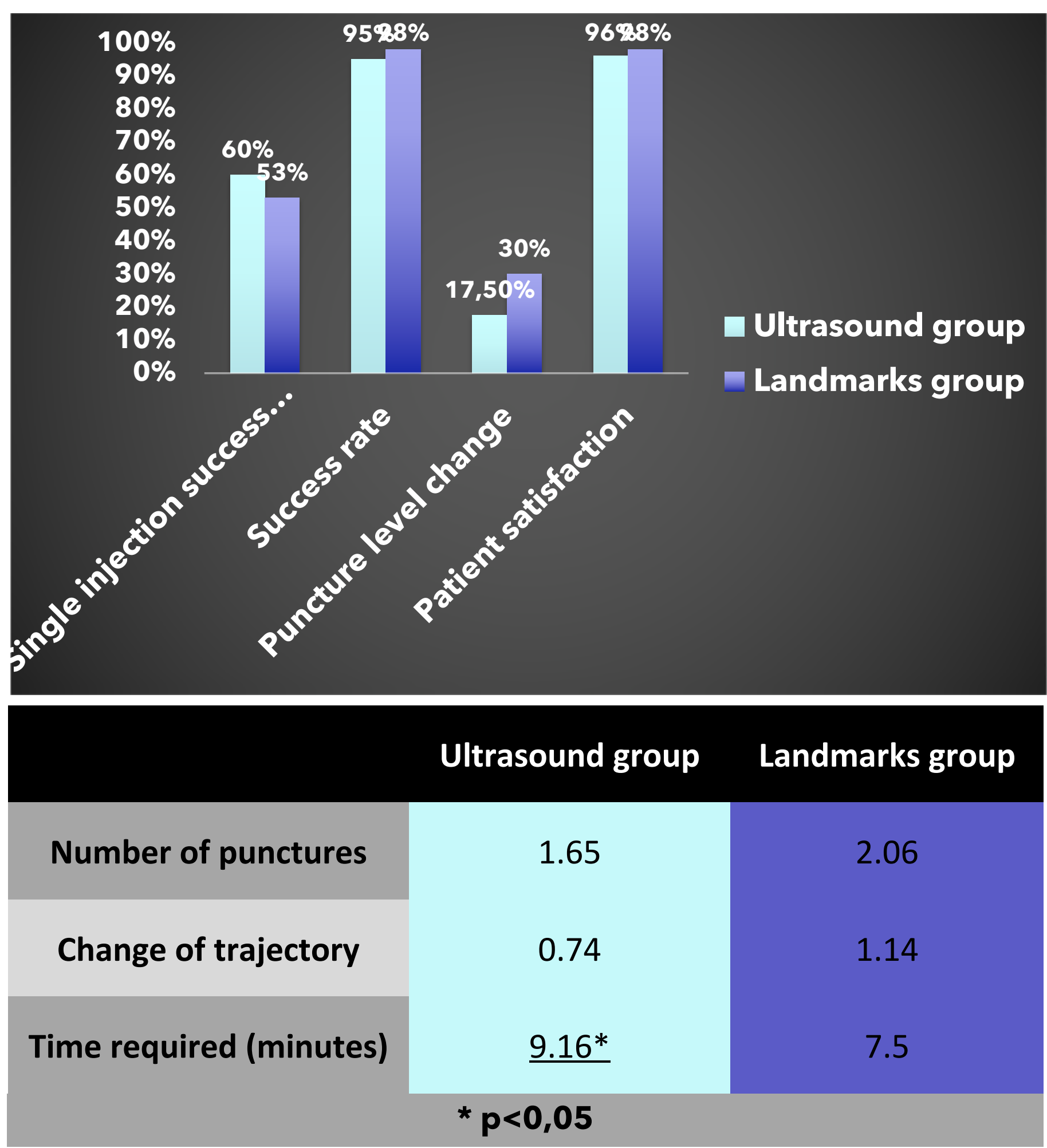

1 Arzola C, Davies S, Rofaeel A, Carvalho JCA. Ultrasound using the transverse approach to the lumbar spine provides reliable landmarks for labor epidurals.

Anesth Analg 2007; 104: 1188-92. 